\title{
A STUDY TO ASSESS THE PREVALENCE OF HIGH RISK PREGNANCY AMONG PRIMI AND MULTI GRAVIDA WOMEN ADMITTED IN SELECTED HOSPITAL AT PUDUCHERRY.
}

\author{
*Mrs. S. Lavanya
}

\begin{abstract}
Pregnancy is one of the most memorable movements in the life of women. Nowadays it is more common to find many antenatal motherscoming under the category called -high risk pregnancyll. Globally considering the incidence rate of high-risk pregnancy keeps on increasing due to many factors like life style pattern, diet and also due to idiopathic causes. ${ }^{3}$ Maternal mortality rate is also higher among high risk pregnancy. The main aim of the study was to assess and compare the prevalence of High Risk Pregnancy among Primi and Multi gravida women. Descriptive research design was adopted for the study. Primi and Multi gravida women who were under high-risk pregnancy and those who fulfill the inclusion criteria were selected as samples. The study finding shows that the prevalence rate of Primi gravida women were higher than Multi gravidwomen.
\end{abstract}

Key words: High-risk pregnancy, Primi gravida, Multi gravid.

\section{Introduction}

Child bearing period is an important precious stage in the life of a woman. There may be many changes occurring within the body in order to bear a creation. All through nine months of hard ships in bearing a child in womb, mother only feel pleasure in having a life within her. ${ }^{2}$ Nowadays it is more common to find many antenatal mothers coming under the categorycalled -Highriskpregnancyllonemaybeannoyed hearing this word high-risk pregnancy. High-risk pregnancy is considered high risk when there is potential complication that could affect the mother or fetus orboth. ${ }^{3}$ There may be many factors that contribute as an etiological factor to occur high-risk pregnancy in primi or multi gravida mothers irrespective of how many deliveries they have undergone. The total percentage of high-risk pregnancy every year in India accounts from 35 percent to 40 percent inall pregnancy. ${ }^{4}$ At present scenario, there is gradual rise in high-risk pregnancy cases due to many contributing factors. Globally considering the incidence rate of high-risk pregnancy keeps on increasing due to many factors like life style pattern, diet and also due to idiopathic causes. To avoid these risky pregnancies it is better to schedule a preconception appointment, be caution when using assisted reproductive technology, seek regular prenatal care, eat healthy diet, gain weight wisely and avoid risky substance. ${ }^{5}$ Health care personnel should contribute on early diagnosis and treatment ${ }^{5-7}$. Nurses should co-ordinate with the other heath team members and develop protocols to prevent complications of high-riskpregnancies ${ }^{8-9}$.

\section{Statement of the Problem}

A study to assess the prevalence of high-risk pregnancy among Primi and Multi gravida 
women admitted in MGMC \& RI from

January to April, 2014.

\section{Objectives}

$>$ To assess the prevalence of high risk pregnancy among Primi and Multi gravida women.

$>$ To compare the prevalence of high risk pregnancy among Primi and Multi gravida women.

$>$ To findout the association between high risk pregnancy among Primi and Multi gravida women with selected demographic variables.

\section{Research Methodology}

A retrospective study approach was used to assess the prevalence of high risk pregnancy among Primi and Multi gravida women who were admitted in MGMC \& RI from January to April 2014. Descriptive research design was adopted for the study. Purposive sampling technique was used to select the sample .Primi and Multi gravida women who were under high-risk pregnancy and those who met the inclusion criteria and got admitted in MGMC \& RI during the period of January to April 2014 were selected as sample. The tool used to collect data is based on objectives of the study to assess the prevalence of high-risk pregnancy among primi and multi gravida women. The tool consists of demographic variables of women who were under high risk pregnancy which includes age, educational qualification, occupation, income, Religion, area of residence, marital status, gravid ,gestational age etc. The data were collected from the available patient records at a period from January to April 2014. Before starting data collection researches obtained consent from the HOD, Dept. of OBG, Medical superintendent and MRD officer to conduct the study. Data was collected from the record based on the demographic variables that were listed out in the study. Descriptive and inferential statistics was used to analysis thedata.

Table: 1 Frequency and percentage distribution of prevalence of women who were under high risk pregnancy and got admitted in mgmc\&ri from january to april 2014.

\begin{tabular}{|c|c|c|c|c|c|}
\hline \multirow{2}{*}{ SL.NO } & $\begin{array}{c}\text { Total No. Of Women } \\
\text { Who Got Admitted In } \\
\text { MGMC \& RI From } \\
\text { January To April 2014 }\end{array}$ & \multicolumn{2}{|c|}{$\begin{array}{c}\text { Total No.Of Women Who } \\
\text { Were Under High Risk } \\
\text { Pregnancy }\end{array}$} & \multicolumn{2}{|c|}{ Prevalence Percentage } \\
\hline \multirow{2}{*}{ A. } & 5078 & Primi & Multi & Primi & Multi \\
\cline { 3 - 6 } & & 102 & 61 & $2 \%$ & $1.2 \%$ \\
\hline
\end{tabular}


FIGURE:1 compare the prevalence of high risk pregnancy between primi and multi gravida women.

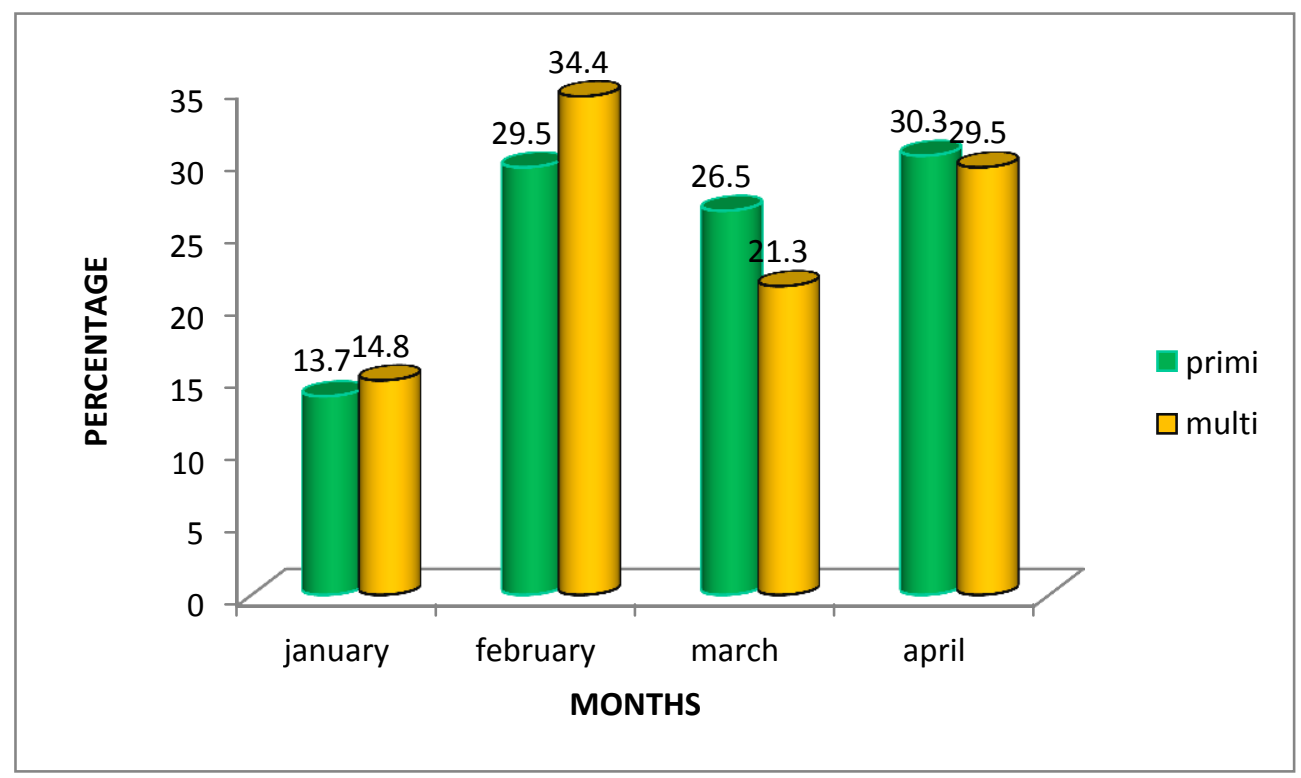

\section{CONCLUSION:}

\section{Major findings:}

- The finding denotes that the total number of women who were under high risk pregnancy were 163 out of 5078 women admitted in MGMC\&RI. Hence the total prevalence of high risk pregnancy from January April 2014 was found to be $3.2 \%$.

- Out of 163 samples, 102 (62.2\%) were Primigravida women, 61(37.4\%) were Multigravida women, the results shows that the prevalence rate were higher among Primigravida than Multigravidawomen.

- There is a significant association between high risk pregnancy and previous bad obstetrical history at the level of $p$ value ( $p$ value $<0.05$ ), because there is no significant association between high risk pregnancy with demographic variables like age of women, educational status, occupation, religion, residence, type of marriage, gestational age and medicationconsumed.
A pregnancy is considered high risk when there is potential complication that could affect the mother, baby or both. High risk pregnancy require management by specialist to help ensure the best outcome for the mother and baby. We can reduce the maternal mortality rate through early identification and management of high risk pregnancy.

\section{Referances}

1. Bennt V Ruth Et .al. A text book formidwives $.12^{\text {th }}$ edition, London, W.B sounders company.(1999)

2. Marilynn marchione. A trubling trend of high risk pregnancy in U.S. 2007(March4).

3. Lisa S.Goldberg $\mathrm{x}$, Heather A Simmons. High risk pregnancy after perinatal loss: understanding the label.2011.pp452-457.

4. Mannisto T. Pressure in pregnancy.2013.

5. Johnson CA. High risk pregnancy \&Hypothyroidism in the bitch.2008 [Dec 06]. 
6. Burronghs . Maternity nursing. $7^{\text {th }}$ edition, Tokyo. W.B squanders' company published, (1997),

7. Dutta.D.C. Text book of Obstetrics $.14^{\text {th }}$ edition, New central book agency Calcutta. (2007)pp631-637
8. Jayneklossner $\mathrm{M}$.Introductory Maternity nursing. $1^{\text {st }}$ edition, London Lippincott William \&wilkins.(2006),

9. Lowder Milk Perry. Maternity \& women's health care $.9^{\text {th }}$ edition, New York. Mosby Elsevier publication,(2007) 